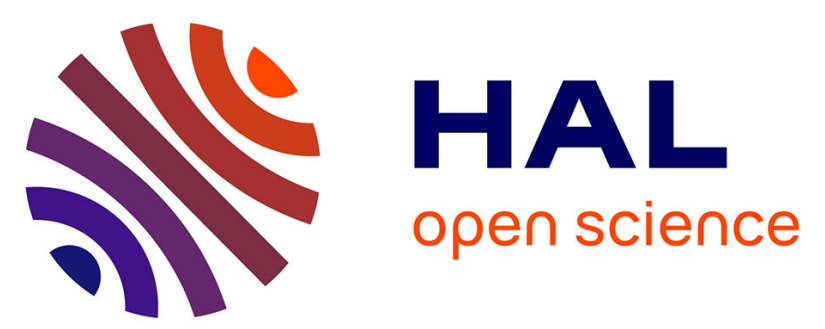

\title{
EFFETS DES TRAITEMENTS THERMIQUES SUR LES PROPRIETES ELECTRIQUES DES CELLULES SOLAIRES A BASE DE SILICIUM POLYCRISTALLIN
}

\author{
E. Courcelle, M. Mesli, J. Muller, D. Salles, P. Siffert
}

\section{- To cite this version:}

E. Courcelle, M. Mesli, J. Muller, D. Salles, P. Siffert. EFFETS DES TRAITEMENTS THERMIQUES SUR LES PROPRIETES ELECTRIQUES DES CELLULES SOLAIRES A BASE DE SILICIUM POLYCRISTALLIN. CNRS International Colloquium on Polycristalline semiconductors, 1982, Perpignan, France. pp.C1-283-C1-288, 10.1051/jphyscol:1982139 . jpa-00221796

\section{HAL Id: jpa-00221796 https://hal.science/jpa-00221796}

Submitted on 1 Jan 1982

HAL is a multi-disciplinary open access archive for the deposit and dissemination of scientific research documents, whether they are published or not. The documents may come from teaching and research institutions in France or abroad, or from public or private research centers.
L'archive ouverte pluridisciplinaire HAL, est destinée au dépôt et à la diffusion de documents scientifiques de niveau recherche, publiés ou non, émanant des établissements d'enseignement et de recherche français ou étrangers, des laboratoires publics ou privés. 
JOURNAL DE PHYSIQQUE

Colloque C1, supplément au nº10, Tome 43, octobre 1982

page $\mathrm{Cl}-283$

\title{
EFFETS DES TRAITEMENTS THERMIQUES SUR LES PROPRIETES ELECTRIQUES DES CELLULES SOLAIRES A BASE DE SILICIUM POLYCRISTALLIN
}

\author{
E. Courcelle, M. Mes1i, J.C. Muller, D. Salles et P. Siffert \\ Centre de Recherches Nuclëaires, Laboratoire de Physique et Applications \\ des Semiconducteurs, (PHASE), 67037 Strasbourg Cedex, France
}

\begin{abstract}
Résumé. - On a étudié l'effete de recuits à haute température sur les caractéristiques électriques à l'obscurité et sous éclairement de cellules solaires à base de silicium polycristallin dont la jonction était préparée à froid par incrustation ionique et recuit par laser pulsé. Dans certains cas des dégradations notables sont observées pour des recuits à $900^{\circ} \mathrm{C}$.
\end{abstract}

Abstract. - The effect of high temperature treatments has been investigated on polycristalline silicon solar cells prepared by a "cold" technology including $\mathrm{PF}_{5}$ ion incrustation and pulsed $\mathrm{YAG}$ laser annealing. In some cases, degradation is observed after heating of $900^{\circ} \mathrm{C}$.

1. Introduction

Le silicium polycristallin tend à remplacer progressivement les monocristaux dens la fabrication des photopiles. La technologie d'élaboration de la jonction reste la même et fait appel à la diffusion thermique de dopants. Or certaines études récentes (1) montrent que ces cycles thermiques peuvent altérer notablement les propriétés du matériau de départ.

Le but de ce travail est d'étudier I'influence des traitements thermiques sur les propriétés électriques et photovoltaïques de quelques polycristaux CGE, tirés par la méthode Bridgmann (2). Pour ne pas modifier les propriétés du matériau de départ, 1 a jonction $\mathrm{P}-\mathrm{N}$ a été réalisée par un procédé à froid utilisant I'incrustation ionique et le recuit par laser pulsé.

\section{Conditions expérinentales}

- Silicium polycristallin

Les cristaux foumis par la CGE ont été tirés par une méthode de Bridgmann modifiée(2) à partir de silicium de qualité électronique. La taille des polycristaux varie suivant l'endroit dans le bloc où l'échantillon est prélevé. La résistivité de ces cristaux de type P évolue également le long du lingot comme indiqué sur la figure 1; elle est plus faible dans la partie supérieure (tranche 11) qu'inférieure (tranche 44). Par ailleurs, des mesures $C(V)$ indiquent que la résistivité évolue également dans la direction perpendiculaire à I'axe de tirage (figure 2). Le dopage est plus important au centre du lingot qu'à la périphérie : cela montre que les courbes d'isorésistivité ont leur concavité toumée vers le haut du lingot.Celles-ci dépendent des conditions de tirage et varient d'un lingot à $l$ 'autre (2).

- Recuit thermique

Le recuit thermique des échantillons est effectué après sciage en tranches de $300 \mu \mathrm{m}$ d'épaisseur et décapage conventionnel dans des conditions proches de celles mises en oeuvre pour la diffusion thermique, soit $900^{\circ} \mathrm{C}$ pendant 1 heure, sous vide (pression : $10^{-6}$ torr). 

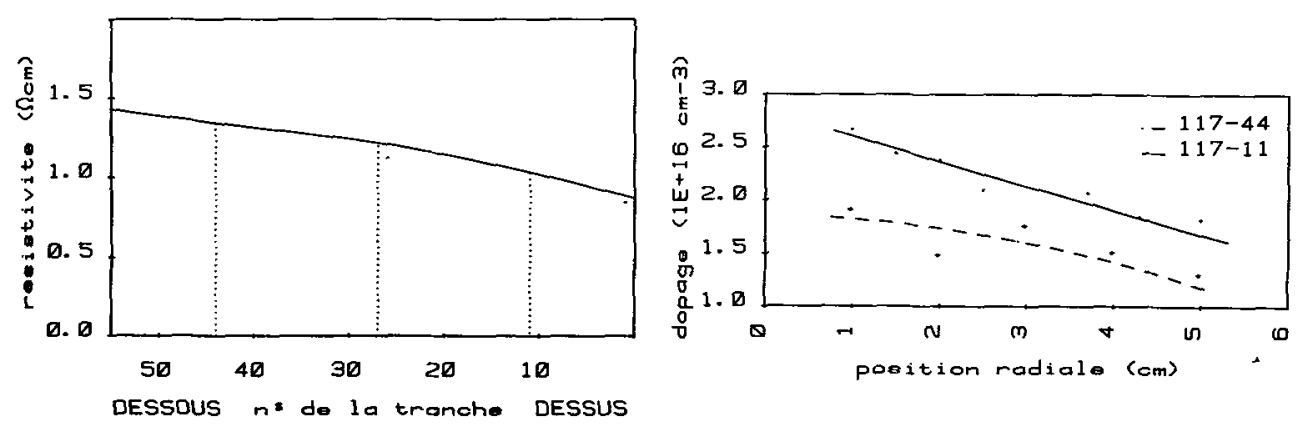

F1g. 1 : Résistivité en fonction

de la hauteur de l'échantilion sur le lingot (courbe fournie par la CGE).

Fie. 2 : Dopage du siliciuri en

fonction de la distance è l'axe de tirage du lingot (mesuré par $C(V)$ ).

\section{- Jonction et contacts}

La jonction $\mathrm{P}-\mathrm{N}$ est réalisée par incrustation ionique à partir de $\mathrm{PF}_{5}$ par le procédé AMI mis au point au laboratoire (3). La tension d'accélération est de $10 \mathrm{keV}$ et la densité de courant ionique de $0,4 \mathrm{~mA} / \mathrm{cm}^{2}$. La dose incrustée est de $5-10^{16} \mathrm{~cm}^{-2}$.

La guérison des domages superficiels et l'incorporátion des dopants au réseau est obtenue par recuit laser à l'aide d'un ensemble "Epitherm" utilisant le balayage d'um laser YAG pulsé ầ haut taux de répétition ( $5 \mathrm{kHz}$ ), émettant des impulsions de durées $100 \mathrm{~ns}$, pour un faisceau de diamètre $100 \mu \mathrm{m}$. La censité d'énergie employée a été de $2,5 \mathrm{~J} / \mathrm{cm}^{2}$ à $0,53 \mathrm{\mu m}$ de longueur d'onde. Au-delà de la jonction, le natériau ne subit pratiquement pas d'échauffement.

Un recuit thermique à $600^{\circ} \mathrm{C}$ permet de guérir les défauts résiduels, notament ceux dûs ì la queue d'implantation non touchée au cours du recuit laser. Des diodes mesa de 2 ou $6 \mathrm{rm}$ de diamètre sont finalernent dégagées aux endroits choisis.

Une métallisation ce $2000 \AA$ d'or assure le contact de la face arrière, alors que la face frontale est munie d'une grille en argent d'épaisseur $3000 \AA$.

\section{Résultats expérinientalix}

Trois types de mesures ont été effectuées, à savoir les caractéristiques I-V et $\mathrm{C}-\mathrm{V}$ à I'obscurité, les propriétés photovoltäịues sous un éclajrement simulé Alil $\left(100 \mathrm{~mW} / \mathrm{cm}^{2}\right)$ et la longueur cie diffusion effective des porteurs minoritaires dans la base, des niesures DLTS. Dans ce dernier cas, on a préféré utiliser des structures Schottky pour ne pas être gênés par d'éventuels défauts dûs au procédé de fabrication de la jonction. Elles ne sont préparées qu'après recuit des échantilions à difrérentes températures.

\section{A. A l'obscurité}

a) Iresures I-V à l'ouscurité

En négligeant l'effet c'es résistances série et de shumt, l'expression du courant peut se mettre sous la forme :

$$
I=1_{\text {or }} \exp \frac{q V}{n_{r} k T}+1_{\text {od }} \exp \frac{q V}{n_{d} k T}
$$


dans laçuelle la première partie est due à la recomibinaison en volume et en surface, la seconde au courant de diffusion. Comme on peut le constater sur la figure 3 , les valeurs du courant à faible polarisation, où le courant de recombinaison domine, sont très dispersées, ce qui résulte du fait que les joìnts de grain et les effets de surface jouent un rôle important, mais aléatoire.

Par contre, la composante de diffusion est très stable d'un échantillon à l'autre, ce qui indique que la jonction est homogène. Nous avons rassemblé sur le tableau I les paramètres cul courant ainsi que ceux mesurés pour du silicium monocristallin préparé dans les mêmes conditions. On notera la très grande différence de densité de courant, ce qui indique la présence d'une grande densité de défauts dans le polycristal.

Fig. 3 : Courbes $I(V)$ à 1 'obscurité pour deux échantillons 117-11 sans recuit thermicue et deux échantillons avec recuit thernique.

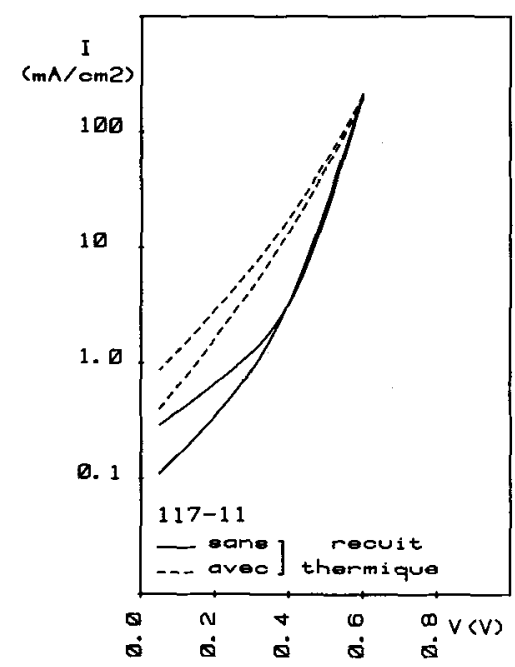

b) Nature des défauts

Afin de confirmer la responsabilité du traitement thermique sur la dégradation des propriétés électriques du matériau, nous avons analysé par DLIS divers échantillons ayant subi des traitements thermiques à 300,700 et $900^{\circ} \mathrm{C}$ pendant $60 \mathrm{~min}$.

La figure 4 montre les spectres obtenus pour les échantilions 11 , 27 et 44 du même lingot : tant que la température ne dépasse pas $700^{\circ} \mathrm{C}$ les spectres DLTS sont plats; par contre à $900^{\circ} \mathrm{C}$ un niveau apparaît à $\mathrm{E}_{\mathrm{V}}+0,33 \mathrm{eV}$ à une concentration

décroissante en passant du 44 (partie inférieure du lingot) au 27 puis au 11. Il pourrait s'agir du centre $K$ (vacance $\mathrm{C}-\mathrm{O}$ ) déjà reporté dans $1 \mathrm{la}$ littérature $(4,5)$.

Fig. 4 : Spectre DLTS. Il apparâ̂t un niveau à l'énergie $\mathrm{E}_{\mathrm{V}}+0,33 \mathrm{eV}$

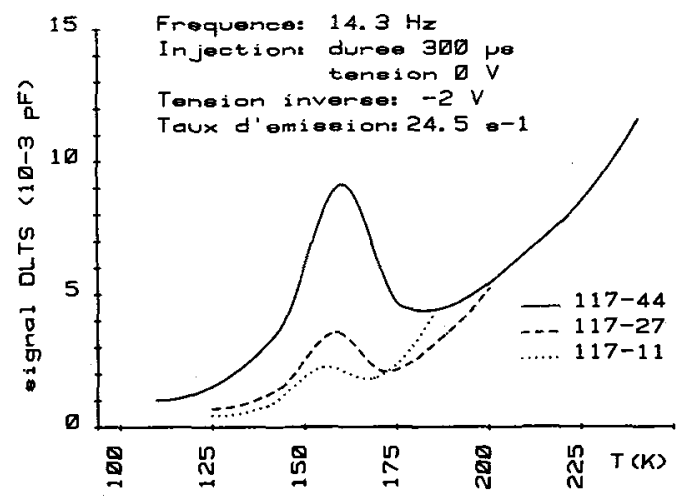
section efficace de capture $\sigma_{\mathrm{p}}=3 \cdot 10^{-15} \mathrm{~cm}^{2}$.

Ce défaut observé en l'absence de porteurs minoritaires (Schottky), joue le rôle de centres de recombinaison dans la jonction. Il détermine la longueur de diffusion et la tension de circuit ouvert. 


\section{B. Sous éclairement AM1}

a) Préalablement au recuit thermique

Nous avons reporté sur le tableau II les meilleurs résultats enregistrés pour les tranches $117-11$ et 44 , comparés à du wacker. On notera que le meilleur rendement est mesuré sur le $n^{\circ} 11$, prélevé à la partie supérieure, puisque à la fois la tension de circuit ouvert et la longueur de diffusion sont meilleures, ce qui est en accord avec les résultats de la DLTS.

Il apparaît également que les échantilions les moins résistifs présentent les meilleures caractéristiques. Ce résultat est lui aussi confirmé par DLTS.

Remarquons que les meilleurs résultats obtenus sur ce matériau sont voisins de ceux mesurés sur du matériau Wacker dont la jonction a été réalisée de la même manière.

b) Après traitement thermique

Le tableau III résume les résultats obtenus sur l'échantillon 11 avant et après recuit thermique. On constate que la tension de circuit ouvert est sensiblement dégradée par le recuit thermique, alors que le courant de court-circuit et la longueur de diffusion sont sensiblement les mêmes.

\section{c) Interprêtation}

La différence de comportement des échantillons réalisés sur la tranche 117-11 et 117-44 pourrait s'expliquer par la présence de dislocations vers le bas du lingot : 'celles-ci sont dues au fait que la croissance ne se fait pas à partir de germes monocristallins, mais de centres de nucléation dispersés sur le fond du creuset. D'autre part, la vitesse de croissance est sensiblement plus grande que dans du silicium monocristallin.

La présence d'un centre de recombinaison observé en DITS pourrait alors s'expliquer par un effet de diffusion de lacunes et d'oxygène vers les dislocations. Cet effet est bien connu dans le silicium monocristallin (6) comme dans le polycristallin, où la diffusion d'oxygène peut aussi se faire vers les joints de grains (1).

Dans cette hypothèse, les dislocations qui jouent un rôle néfaste en augmentant le courant inverse et en faisant baisser la tension de circuit ouvert, corme les expériences réalisées sur l'échantillon 117-44 le montrent, devraient introduire des niveaux visibles par DLTS sur les spectres effectués sur des échantillons $n$ 'ayant pas subi de recuit thermique. Les mesures que nous avons réalisées nous permettent seulement d'affirmer que le centre $K n^{\prime}$ existe pas pour des recuits thermiques à des températures inférieures à $900^{\circ} \mathrm{C}$. Par ailleurs, il n'est pas exclu que d'autres pics soient présents à des concentrations inférieures à notre seuil de détection. $D^{\prime}$ autres expériences devraient nous permettre de lever cette ambiguité.

\section{Conclusion}

Cette étude a permis de montrer que les résultats obtenus sur le silicium polycristallin CGE dépendent fortement de l'endroit dans le lingot où est prélevé 1 'échantillon. Par ailleurs, les traitements thermiques ont des effets non négligeables, dégradant le rendement de conversion photovoltaïque. Il convient donc d'être prudent lors de la comparaison des divers polycristaux, car la méthode d'élaboration de la cellule peut jouer un rôle non négligeable.

\section{Remerciements}

Nous remercions M. DUMAS et FAILY (CGE) qui ont bien voulu mettre à notre disposition les échantilions utilisés dans ce travail, ainsi que Mlle AMANN qui a préparé les diodes. 


\section{Références}

(1) RUSSEIL P.E., HERRINGTON C.R., BURKE D.E. and HOLLOWAY P.H.

The effect of heat treatment on grain boundary properties in cast polycristalline silicon. In Proc. Materials Research society Conf. de Boston "Grain boundaries in Semiconductors" pp. 185, 191 (Nov. 1981).

(2) FALLY J.

Rapport interne CGE, non publié, Juin 1981.

Etude d'élaboration de silicium semi-cristallin à structure basaltique en tant que matériau pour photopiles solaires.

(3) MULLER J.C., PONPON J.P., GROB J.J. and SIFFERT P. in Proc. European Photovol taïc Solar Energy. Conf. D. Reidel Ed. (Luxembourg) pp. 897-909, sept. 1977.

(4) CARTER J,R.

Jr. IEEE Trans. on Nuclear Sc.13, 6 (1966).

(5) CARTER J.R.

Joumal of Chemistry and Physics of Solids 27 (1966) p. 913.

(6) MAGEE T.J., LEUNG C., KAWAYOSHI, FURMAN B., EVANS C.A., DAY D.S.

Redistribution of oxygen within damage regions of boron-implanted silicon Appl. Phys. Lett. 39 (3), august 1981.

\section{Tableau 1}

\begin{tabular}{|l|c|c|c|c|}
\hline & $1_{\text {or }}\left(\mu \mathrm{A} / \mathrm{cm}^{2}\right)$ & $n_{r}$ & $l_{\text {od }}\left(\mu \mathrm{A} / \mathrm{cm}^{2}\right)$ & $n_{d}$ \\
\hline $\begin{array}{l}117-11 \\
\text { sans recuit th. }\end{array}$ & 75,4 & 5,4 & 0,153 & 1,7 \\
\hline $\begin{array}{l}117-11 \\
\text { avec recuit th. }\end{array}$ & 247 & 4,58 & 7,79 & 2,44 \\
\hline Si monocristallin & 0,225 & 2,4 & $3,1,10^{-3}$ & 1,38 \\
\hline
\end{tabular}

Tableau 11. (Valeurs mesurées sans couche anti-reflet)

\begin{tabular}{|l|c|c|c|c|c|}
\hline & $V_{c o}(\mathrm{mV})$ & $I_{c c}\left(\mathrm{~mA} / \mathrm{cm}^{2}\right)$ & $F F$ & $\eta \%$ & $L_{n}(\mu \mathrm{m})$ \\
\hline $117-11$ & 505 & 23 & 0,67 & 7,8 & 37 \\
\hline $117-44$ & 480 & 20 & 0,64 & 6,1 & 16 \\
\hline Wacker & 505 & 20,6 & 0,7 & 7,3 & 35 \\
\hline
\end{tabular}


Tableau 111. (Valeurs mesurées sans couche anti-reflet)

\begin{tabular}{|l|c|c|c|c|c|}
\hline & $V_{c o}(\mathrm{mV})$ & $1_{c c}\left(\mathrm{~mA} / \mathrm{cm}^{2}\right.$ & $F F$ & $\eta \mathrm{L}_{\mathrm{n}}(\mu \mathrm{m})$ \\
\hline $\begin{array}{l}117-11 \\
\text { SANS recuit th. }\end{array}$ & 505 & 21,2 & 0,65 & 7 & 30 \\
\hline $\begin{array}{l}117-11 \\
\text { AVEC recuit th. }\end{array}$ & 475 & 21 & 0,62 & 6,2 & 25 \\
\hline
\end{tabular}

